\title{
Probing momentum distributions in magnetic tunnel junctions via hot-electron decay
}

\author{
R. Jansen, ${ }^{\text {a) }}$ T. Banerjee, B. G. Park, and J. C. Lodder \\ $\mathrm{MESA}^{+}$Institute for Nanotechnology, University of Twente, 7500 AE Enschede, The Netherlands
}

(Received 3 April 2007; accepted 11 April 2007; published online 8 May 2007)

\begin{abstract}
The tunnel momentum distribution in a (magnetic) tunnel junction is probed by analyzing the decay of the hot electrons in the Co metal anode after tunneling, using a three-terminal transistor structure in which the hot-electron attenuation is sensitive to the tunnel momentum distribution. Solid state amorphous $\mathrm{Al}_{2} \mathrm{O}_{3}$ barriers and the vacuum barrier of a scanning tunneling microscope are compared. For the former the attenuation length in nominally the same Co is strikingly larger (factor of 2), implying a more isotropic tunnel momentum distribution for $\mathrm{Al}_{2} \mathrm{O}_{3}$ barriers. (C) 2007 American Institute of Physics. [DOI: 10.1063/1.2737128]
\end{abstract}

Tunneling is a fundamental quantum mechanical process that is applied in numerous electronic devices. In the last decade, the role of electron spin in tunneling has attracted widespread attention, ${ }^{1-7}$ not in the least because of the prospective applications in an ever increasing diversity of spintronic structures (magnetic sensors, memory, spin-transfer torque devices, and spin-injection into semiconductors). Nevertheless, the understanding of spin-dependent tunneling is still incomplete despite significant progress. There is so far no widely applicable approach to reliably predict the sign and magnitude of the tunneling magnetoresistance (TMR) from the electronic structure of the materials used to construct a magnetic tunnel junction (MTJ).

One reason is that for the critical interfacial region of a MTJ, precise enough experimental information about the structural, chemical, and magnetic properties is often lacking. In addition, theoretical calculations necessarily make assumptions about the atomic arrangement at the ferromagnetinsulator interface but often neglect disorder, while amorphous barriers are notoriously difficult to handle. Coherent tunneling is often assumed, in which states on either side of the tunnel barrier couple to evanescent states in the barrier and allow coherent transfer of electrons without momentum scattering. In such cases, arguably applicable to crystalline tunneling junctions using, e.g., $\mathrm{MgO}$ or $\mathrm{SrTiO}_{3}$ barriers, symmetry arguments can be used to determine which states are likely to contribute to the tunnel current. ${ }^{8-10}$ However, in general, solid-state tunnel barriers (such as frequently employed amorphous $\mathrm{Al}_{2} \mathrm{O}_{3}$ ) contain a certain fraction of defects within the barrier, as well as interfacial roughness and disorder. As a result, symmetry arguments are relaxed and tunneling can be accompanied by momentum scattering, ${ }^{11,12}$ thereby connecting states for which coherent tunneling is not possible. The interest in this has recently been revived with the advent ${ }^{4,5}$ of crystalline $\mathrm{MgO}$ based MTJs, where defects limit the TMR (Ref. 13) and may also play a role in the spin-transfer torque ${ }^{6}$ that can be created using injection of a spin-polarized tunnel current.

Given the ubiquity of magnetic tunnel junctions, it appears pertinent to determine the extent of momentum scattering in tunneling. For an understanding of (spin-dependent) tunneling and a meaningful comparison with model calcula-

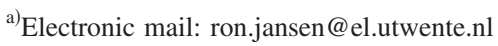

tions, such information is indispensable. However, it cannot be extracted directly from the TMR.

Here we provide experimental information about the extent of momentum scattering in a tunnel junction. We extract this from an analysis of the decay of the hot electrons in the Co metal anode after tunneling, using a three-terminal transistor geometry. We compare two different types of barriers, namely, amorphous $\mathrm{Al}_{2} \mathrm{O}_{3}$ and vacuum tunnel barriers, as often used, respectively, in MTJs or scanning tunneling microscopy (STM) based experiments. The results demonstrate that the tunneling momentum distribution is much more isotropic for tunneling via amorphous $\mathrm{Al}_{2} \mathrm{O}_{3}$. Tunneling across a vacuum barrier, in which scattering sites are absent, produces a more forward-focused momentum distribution dominated by electrons with small momentum component parallel to the surface. Simultaneously, the results show that the decay length of hot electrons in $\mathrm{Co}$ is sensitive to the momentum distribution of the injected hot-electron current.

The experiment uses a tunnel junction incorporated into a three-terminal hot-electron transistor geometry, consisting of the tunnel emitter, a metallic base, and a semiconductor collector. Two implementations will be used: (i) a solid-state magnetic tunnel transistor (MTT) ${ }^{14-16}$ with $\mathrm{Ni}_{80} \mathrm{Fe}_{20} / \mathrm{Al}_{2} \mathrm{O}_{3} / \mathrm{Co} / \mathrm{Au} / \mathrm{Si}$ structure, and (ii) ballistic electron emission microscopy (BEEM) ${ }^{17,18}$ using a structure of tip/vacuum/Au/Co/Au/Si. In both cases, the $\mathrm{Co} / \mathrm{Au} / \mathrm{Si}$ base/collector part is used to measure the transmission of the hot electrons after their injection into the Co base by tunneling from the emitter. Measuring the transmission as a function of the Co base thickness yields the hot-electron attenuation length. Although MTT and BEEM are based on nonequilibrium electrons whose decay channels include inelastic scattering, the collection across a Au/Si metalsemiconductor Schottky barrier imposes restrictions not only on the energy but also on the momentum of the hot electrons. ${ }^{16,17}$ The latter is often simplified in a free electron picture in terms of a critical angle of incidence defining a momentum collection cone. ${ }^{17}$ More rigorously, the momentum of the hot electrons has to match that of the available states in the conduction band of the $n$-type Si collector. Consequently, the hot-electron transmission and attenuation length of the Co base is sensitive to the momentum distribution of the hot-electron current as injected by tunneling across the $\mathrm{Al}_{2} \mathrm{O}_{3}$ or vacuum tunnel barrier. 


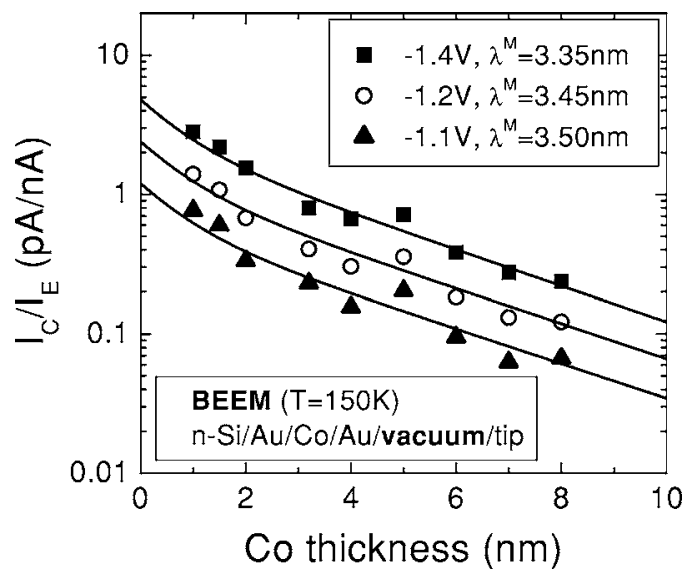

FIG. 1. Hot-electron attenuation in Co measured by BEEM for hot electrons injected by tunneling across a vacuum barrier at the tunnel bias voltages indicated. $T=150 \mathrm{~K}$.

The metal layers for MTT and BEEM studies are fabricated on identical H-terminated $n$-type $\mathrm{Si}(100)$ wafers using thermal evaporation in the same molecular beam epitaxy system at a base pressure of $10^{-10}$ mbar. First, an $8 \mathrm{~nm}$ Au layer was evaporated to form a Au/Si Schottky barrier, onto which the Co base layers of thickness $t_{\mathrm{Co}}$ between 1 and $9 \mathrm{~nm}$ are grown. For the BEEM samples the Co layer was capped with a $3 \mathrm{~nm}$ Au layer to provide a chemically inert surface for $e x$ situ sample transfer to the ultrahigh vacuum BEEM system. BEEM data are obtained at $150 \mathrm{~K}$ using PtIr metal tips. Details of the BEEM measurement have been described elsewhere. ${ }^{19}$ For the MTT samples, an $\mathrm{Al}_{2} \mathrm{O}_{3}$ barrier was created directly on the Co base by deposition of metal Al $(1.8 \mathrm{~nm})$ followed by plasma oxidation. Details as well as the processing into MTT devices have been described previously. ${ }^{15}$ The MTTs showed hot-electron magnetocurrent ${ }^{15}$ of up to $105 \%$ at $100 \mathrm{~K}$, while the TMR of the $\mathrm{Ni}_{80} \mathrm{Fe}_{20} / \mathrm{Al}_{2} \mathrm{O}_{3} /$ Co junctions was $30 \%-32 \%$ at $100 \mathrm{~K}$, indicating good MTJs.

Data for the BEEM technique are shown in Fig. 1, where the hot-electron transmission is given as the ratio of the current $I_{C}$ transmitted into the Si collector and the emitter tunnel current $I_{E}$ injected from the STM tip across the vacuum gap. We observe an exponential attenuation with a typical attenuation length of 3.3 to $3.5 \mathrm{~nm}$. At small $t_{\mathrm{Co}}$ the transmission deviates from the exponential decay because minority spin hot electrons still contribute. Owing to their short attenuation length $(<1 \mathrm{~nm}),{ }^{18}$ these no longer contribute beyond $t_{\mathrm{Co}}$ of about $2 \mathrm{~nm}$, where the attenuation length represents the majority spin hot electrons in Co.

Results for the $\mathrm{Al}_{2} \mathrm{O}_{3}$-based MTT are shown in Fig. 2. Again we observe an exponential attenuation of the hotelectron transmission with increasing $t_{\mathrm{Co}}$. However, the attenuation lengths are $6.7-6.9 \mathrm{~nm}$, which is significantly larger than observed for tunnel injection across a vacuum gap in BEEM. The extracted attenuation lengths in Co versus tunnel bias (or hot-electron energy) are given in Fig. 3 for the MTT and BEEM structures. The attenuation length obtained with the MTT is distinctly larger, by about a factor of 2, over the full energy range. Thus, the hot-electron attenuation length after tunnel injection into nominally the same Co depends significantly on the type of tunnel barrier.

Since the measurements are performed at slightly different temperature $(100 \mathrm{~K}$ for the MTT versus $150 \mathrm{~K}$ for Downloaded 09 May 2007 to 130.89 .148 .36. Redistribution subject

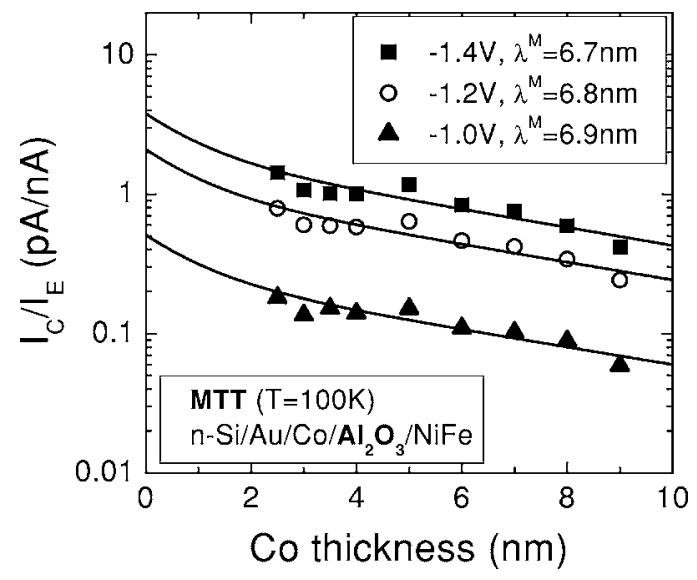

FIG. 2. Hot-electron attenuation in Co measured in an MTT for hot electrons injected by tunneling across an amorphous $\mathrm{Al}_{2} \mathrm{O}_{3}$ barrier at the tunnel bias voltages indicated. $T=100 \mathrm{~K}$.

BEEM), we studied how the hot-electron transmission in Co depends on temperature for the MTT (see Fig. 4). While some variation of the transmission with temperature is observed, the effect is very limited, and in terms of attenuation lengths it corresponds to a change of at best a few angstroms between 100 and $150 \mathrm{~K}$. Thus, the difference in attenuation lengths between MTT and BEEM is not due to the measurement temperature.

Since the experiments use identical Co base layers, the difference in attenuation length is due to the different momentum distribution of the hot-electron current injected by tunneling across $\mathrm{Al}_{2} \mathrm{O}_{3}$ and vacuum barriers. For the latter, if the tunnel momentum distribution is sharply forwardfocused and dominated by electrons with small wave vector parallel to the surface, most of the electrons injected into the Co base satisfy the momentum selection requirements for subsequent transmission across the $\mathrm{Au} / \mathrm{Si}$ interface. However, in this situation the collector current is very sensitive to elastic scattering during transport through the Co base. The polycrystalline Co layers contain defects, grain boundaries, and stacking faults giving rise to momentum scattering. This broadens the momentum distribution of the hot electrons, increasing the fraction of hot electrons that do not have the proper momentum for transmission into the $\mathrm{Si}$. Such a contribution of elastic scattering to the hot-electron attenuation in the Co base leads to short decay lengths, as observed for the BEEM samples.

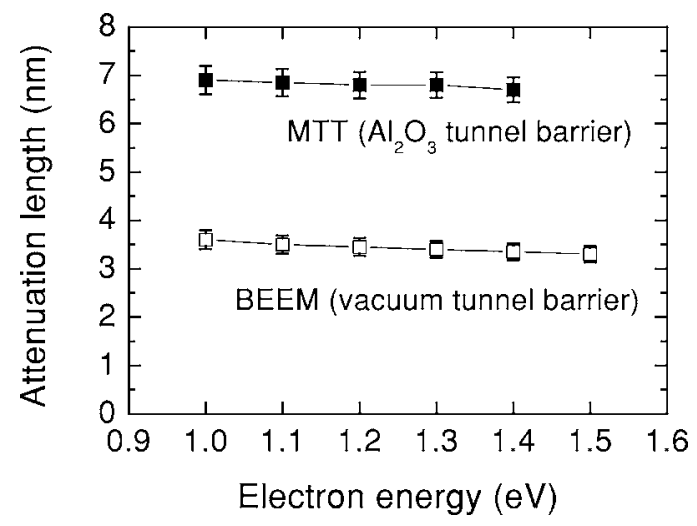

FIG. 3. Hot-electron attenuation lengths vs energy in the Co anode after tunnel injection via a vacuum barrier (extracted from BEEM) or an amorphous $\mathrm{Al}_{2} \mathrm{O}_{3}$ tunnel barrier (extracted from MTT).

o AIP license or copyright, see http://apl.aip.org/apl/copyright.jsp 


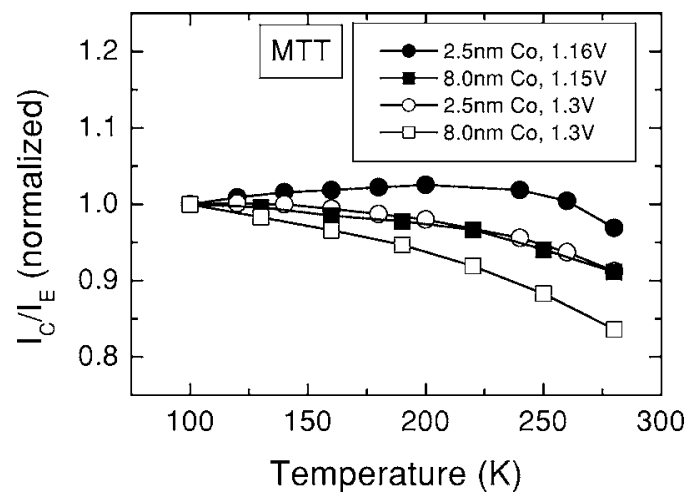

FIG. 4. Temperature variation of hot-electron transmission in Co measured in a MTT for Co base thickness of $2.5 \mathrm{~nm}$ (circles) and $8.0 \mathrm{~nm}$ (squares) at tunnel bias voltages as indicated. Data are normalized to unity at $100 \mathrm{~K}$.

The results with $\mathrm{Al}_{2} \mathrm{O}_{3}$ barriers can be explained if tunneling across amorphous $\mathrm{Al}_{2} \mathrm{O}_{3}$ yields a significantly broader momentum distribution compared to vacuum tunneling. If the tunnel barrier itself already provides significant momentum scattering and the hot electrons are injected into the Co base with an isotropic momentum distribution, then elastic scattering in the Co base has little further effect and does not contribute to the attenuation. The resulting attenuation length becomes larger and is limited by inelastic scattering only. Therefore, the results imply that tunneling across an amorphous $\mathrm{Al}_{2} \mathrm{O}_{3}$ barrier yields a significantly broader momentum distribution compared to vacuum tunneling. The difference cannot be explained by a different energy distribution for the two tunnel barriers, since the attenuation lengths vary only weakly with energy (Fig. 3).

Thus, we have shown that a measurement of the hotelectron decay length in the anode of a MTJ provides information about the extent of momentum scattering in a MTJ. The method can be applied to a variety of materials, including crystalline (e.g., $\mathrm{MgO}$ ) based tunnel junctions that can be grown on lattice matched III-V semiconductors. ${ }^{20}$ Quantification of the extent of momentum scattering is possible using a detailed hot-electron transport model that includes the injected momentum distribution and elastic scattering, as is already available for the spin-valve transistor. ${ }^{21}$
The authors thank E. Haq for his input at the early stages of this work. They acknowledge financial support from the NWO-VIDI program and the NanoNed program coordinated by the Dutch Ministry of Economic Affairs.

${ }^{1}$ J. S. Moodera, L. R. Kinder, T. M. Wong, and R. Meservey, Phys. Rev. Lett. 74, 3273 (1995).

${ }^{2}$ S. S. P. Parkin, X. Jiang, C. Kaiser, A. Panchula, K. Roche, and M. Samant, Proc. IEEE 91, 661 (2003).

${ }^{3}$ I. Zutić, J. Fabian, and S. Das Sarma, Rev. Mod. Phys. 76, 323 (2004).

${ }^{4}$ S. S. P. Parkin, C. Kaiser, A. Panchula, P. M. Rice, B. Hughes, M. Samant, and S.-H. Yang, Nat. Mater. 3, 862 (2004).

${ }^{5}$ S. Yuasa, T. Nagahama, A. Fukushima, Y. Suzuki, and K. Ando, Nat. Mater. 3, 868 (2004).

${ }^{6}$ Y. Huai, F. Albert, P. Nguyen, M. Pakala, and T. Valet, Appl. Phys. Lett. 84, 3118 (2004); G. D. Fuchs, N. C. Emley, I. N. Krivorotov, P. M. Braganca, E. M. Ryan, S. I. Kiselev, J. C. Sankey, D. C. Ralph, R. A. Buhrman, and J. Katine, Appl. Phys. Lett. 85, 1205 (2004).

${ }^{7}$ V. F. Motsnyi, J. De Boeck, J. Das, W. Van Roy, G. Borghs, E. Goovaerts, and V. I. Safarov, Appl. Phys. Lett. 81, 265 (2002); B. T. Jonker, Proc. IEEE 91, 727 (2003); S. A. Crooker, M. Furis, X. Lou, C. Adelmann, D. L. Smith, C. J. Palmstrøm, and P. A. Crowell, Science 309, 2191 (2005); B. C. Min, K. Motohashi, J. C. Lodder, and R. Jansen, Nat. Mater. 5, 817 (2006).

${ }^{8}$ W. H. Butler, X.-G. Zhang, T. C. Schulthess, and J. M. MacLaren, Phys. Rev. B 63, 054416 (2001).

${ }^{9}$ J. Mathon and A. Umerski, Phys. Rev. B 63, 220403 (2001).

${ }^{10}$ J. P. Velev, K. D. Belashchenko, D. A. Stewart, M. van Schilfgaarde, S. S. Jaswal, and E. Y. Tsymbal, Phys. Rev. Lett. 95, 216601 (2005).

${ }^{11}$ A. M. Bratkovsky, Phys. Rev. B 56, 2344 (1997).

${ }^{12}$ J. Zhang and R. M. White, J. Appl. Phys. 83, 6512 (1998); R. Jansen and J. S. Moodera, Phys. Rev. B 61, 9047 (2000).

${ }^{13}$ J. P. Velev, K. D. Belashchenko, S. S. Jaswal, and E. Y. Tsymbal, Appl. Phys. Lett. 90, 072502 (2007).

${ }^{14}$ S. van Dijken, X. Jiang, and S. S. P. Parkin, Phys. Rev. B 66, 094417 (2002).

${ }^{15}$ B. G. Park, T. Banerjee, B. C. Min, J. G. M. Sanderink, J. C. Lodder, and R. Jansen, J. Appl. Phys. 98, 103701 (2005).

${ }^{16}$ R. Jansen, J. Phys. D: Appl. Phys. 36, R289 (2003).

${ }^{17}$ W. J. Kaiser and L. D. Bell, Phys. Rev. Lett. 60, 1406 (1988).

${ }^{18}$ W. H. Rippard and R. A. Buhrman, Phys. Rev. Lett. 84, 971 (2000).

${ }^{19}$ E. Haq, H. Gokcan, T. Banerjee, F. M. Postma, M. H. Siekman, R. Jansen, and J. C. Lodder, J. Appl. Phys. 95, 6930 (2004); T. Banerjee, E. Haq, M. H. Siekman, J. C. Lodder, and R. Jansen, Phys. Rev. Lett. 94, 027204 (2005).

${ }^{20}$ X. Jiang, R. Wang, R. M. Shelby, R. M. Macfarlane, S. R. Bank, J. S. Harris, and S. S. P. Parkin, Phys. Rev. Lett. 94, 056601 (2005).

${ }^{21}$ R. Vlutters, O. M. J. van 't Erve, R. Jansen, S. D. Kim, J. C. Lodder, A. Vedyayev, and B. Dieny, Phys. Rev. B 65, 024416 (2002). 